\title{
Termoplastia bronquial
}

\section{Bronchial thermoplasty}

Olivia SÁnChez CABRAL ${ }^{1}$; SEbastián FERnÁNDEZ-BuSSY²

\section{Resumen}

El asma es una enfermedad de las vías respiratorias, caracterizada por inflamación crónica, hiperreactividad bronquial y obstrucción variable al flujo aéreo, provocado por una variedad de estímulos, incluyendo los alérgicos, los infecciosos, entre otros. La termoplastia bronquial es un tratamiento innovador que consiste en la aplicación de energía de radiofrecuencia a las vías aéreas proximales para la ablación selectiva al músculo liso peribronquial. Es la primera terapia de intervención no farmacológica aprobada por la Administración de Drogas y Alimentos para asma grave. En vista de que se trata de una terapia basada en un dispositivo, la evaluación global de riesgo-beneficio difiere de la de productos farmacéuticos; los aspectos de seguridad, requisitos reglamentarios, diseños de los estudios y evaluación de la magnitud del efecto pueden ser diferentes. Los mecanismos de acción y el tipo de pacientes que se benefician de la termoplastia bronquial son los retos más importantes para el futuro.

Palabras clave: asma grave, termoplastia bronquial, neumología intervencionista.

\begin{abstract}
Asthma is a disease of the airways that is characterized by chronic inflammation, bronchial hyperreactivity, and variable airflow obstruction, triggered y a variety of stimuli, including, among others, allergic and infectious conditions. Bronchial thermoplasty is an innovative treatment carried out by applying radiofrequency energy to the proximal airways, in order to perform selective ablation of peribronchial muscle. It is the first non-pharmacologic interventional therapy approved by the Food and Drug Administration for severe asthma. Since it is a device-based therapy, its overall risk-benefit evaluation is different from that of pharmacologic products; aspects concerning safety, statutory requirements, study design, and assessment of the magnitude of its effects can be different. The mechanisms of action and the type of patients who could benefit from bronchial thermoplasty are the most important challenges for the future.
\end{abstract}

Keywords: severe asthma, bronchial thermoplasty, interventional pulmonology.

\section{Introducción}

La farmacoterapia para el asma consiste en beta agonistas de acción corta y prolongada, modificadores de los leucotrienos y esteroides inhalados y orales. La Sociedad Americana Torácica, define a los pacientes con asma refractaria como aquellos quienes requieren esteroides orales por más del $50 \%$ al año o altas dosis de esteroides inhalados con el objetivo de obtener un control. En la actualidad, el medicamento aprobado para el asma alérgica en pacientes no controlados con esteroides orales o inhalados, es el anticuerpo antiinmunoglobulina E omalizumab. Su administración se hace cada dos semanas, es costoso para algunos pacientes y proporciona control solo a un subgrupo con asma alérgica. Entre tanto, la termoplastia bronquial fue aprobada por la Administración de Alimentos y Drogas (FDA, sigla en inglés) en Estados Unidos, en abril de 2010, para pacientes mayores de 18 años, con asma grave y persistente no
(1) Neumología y Broncoscopia, Instituto Nacional de Enfermedades Respiratorias (INER), Ciudad de México, México.

(2) Director de Neumología Intervencionista, Clínica Alemana de Santiago, Chile.

Correspondencia: Sebastián Fernández Bussy. Correo electrónico: neumologia@ alemana.cl

Recibido: 10/11/14, Aceptado: 10/12/14 
controlada con esteroides inhalados y medicamentos beta agonistas de acción prolongada (1). Los programas de desarrollo clínico consisten principalmente en tres ensayos clínicos aleatorizados y controlados con asma moderada a grave que se analizarán más adelante. Esta revisión resume la información acerca del mecanismo de acción, el procedimiento, la eficacia, la seguridad y la selección de los pacientes, con miras entender mejor el camino a seguir en esta prometedora terapia.

\section{Procedimiento}

La masa del músculo liso se incrementa considerablemente en pacientes asmáticos en comparación con los controles sanos, con lo cual contribuye a la inflamación crónica y remodelación del asma grave. De esto se deduce que una reducción de la masa del músculo liso bronquial, puede aliviar potencialmente los síntomas y quizás disminuir la remodelación (2). La termoplastia bronquial es un procedimiento de broncoscopia intervencionista para el tratamiento de pacientes con asma grave, no controlados. Su realización requiere la identificación precisa y minuciosa de la anatomía de la vía aérea, además de su aplicación por parte de un broncoscopista con experiencia en un centro de salud con un seguimiento clínico apropiado y con capacidad para hacer frente a las posibles complicaciones posteriores a la realización del procedimiento. Mayse y colaboradores $(3,4)$ describieron la evaluación y el seguimiento adecuado del paciente antes, durante y después de este procedimiento.

\section{Mecanismo de acción}

La vía aérea distal (menor a 2-3 mm de diámetro) representa el 10\% del total de la resistencia en una persona normal. Sin embargo, en los asmáticos existe una inflamación continua de toda la vía aérea, desde la proximal a la más distal. Varios mecanismos de acción explican los efectos benéficos de la termoplastia bronquial. Debido a un aumento del músculo liso de las vías respiratorias, el primer y mejor mecanismo documentado, es la reducción del músculo liso de la pared bronquial evaluado en un modelo canino $(5,6)$, aunque la disminución de la hiperreactividad en pacientes asmáticos después de la termoplastia bronquial ha sido inconsistente. Un segundo mecanismo podría implicar un efecto sobre las propiedades contráctiles del músculo liso bronquial o un endurecimiento de la pared bronquial a la que se une el músculo. Se han investigado los efectos de la aplicación de diferentes temperaturas en la contracción in vitro del músculo liso bovino; la contracción inducida por acetilcolina se inhibió a temperaturas superiores a $\operatorname{los} 55^{\circ} \mathrm{C}$, mientras que la relajación medida por un betaagonista 2 , no se vio afectada (7). No obstante, no está claro si los fenómenos observados en la respuesta aguda puedan persistir a largo plazo in vivo. Otros mecanismos posibles incluyen la secreción de mediadores inflamatorios por las células de músculo liso y cambios en el epitelio bronquial, las terminaciones nerviosas o la función de las células inflamatorias que infiltran la mucosa.

\section{Equipo}

Se utiliza el sistema de termoplastia bronquial Alair (figura 1). El catéter es un dispositivo único, de un solo uso, largo, flexible con un conjunto de electrodos expandibles unidos en un extremo y un asa de despliegue en el otro. Este se introduce a través del canal de trabajo de un broncoscopio estándar, idealmente 4,9 $\mathrm{mm}$ hasta $5,2 \mathrm{~mm}$ de diámetro externo y un canal de trabajo de $2 \mathrm{~mm}$ como mínimo. El conjunto de electrodos se expande para ponerse en contacto con la pared de las vías respiratorias y luego se activa para suministrar energía eléctrica de radiofrecuencia. El controlador de radiofrecuencia es un instrumento electrónico que genera energía controlada. Como la energía eléctrica es transferida desde el electrodo al tejido, la resistencia hace que la energía eléctrica se convierta en energía térmica. El controlador proporciona la intensidad y la duración correcta de energía para el tratamiento a $65^{\circ} \mathrm{C}$, lo que reduce la cantidad de tejido muscular liso de la vía aérea, al tiempo que minimiza los daños colaterales a otras 
estructuras de las vías respiratorias de soporte. El controlador también supervisa el sistema para garantizar la coaptación correcta del catéter a la vía aérea y es cuando la energía eléctrica se transmite. Si no se cuenta con un buen contacto con la pared de la vía aérea, el panel frontal notifica para reubicar los electrodos en contacto adecuado. Para completar el circuito se fija en el paciente un electrodo estándar de retorno tipo gel (3).

\section{Procedimiento}

El procedimiento de termoplastia bronquial se efectúa durante tres sesiones de broncoscopia flexible con sedación o anestesia general, cada una separada por tres a cuatro semanas, para disminuir el riesgo de inducir una exacerbación del asma o edema de la vía respiratoria que pudiera incrementarse de llevarse a cabo en una sola sesión o en un intervalo menor. Se prescribe prednisona $50 \mathrm{mg} /$ día durante los tres días previos al procedimiento, el día del procedimiento y el día posterior. Además se realiza una nebulización con salbutamol previo al procedimiento e inmediatamente posterior al mismo. Se utiliza glicopirrolato o atropina antes del procedimiento, con el fin de disminuir la cantidad de secreciones. Se hace una espirometría con broncodilatador para verificar el estado basal inicial y luego revaluar con nueva espirometría 2 a 4 horas luego de la termoplastia. Durante la primera broncoscopia se trata el lóbulo inferior derecho, en la segunda, el lóbulo inferior izquierdo y en la tercera y última, ambos lóbulos superiores (figura 2) (8). Dada la posibilidad de que se presente síndrome de lóbulo medio debido a que es más largo y estrecho, éste no se trata.

Se realiza una exploración de la vía aérea para evaluar la posible impactación de moco o cicatrización, asegurando una curación correcta. Si las áreas tratadas no han curado, se aplaza el

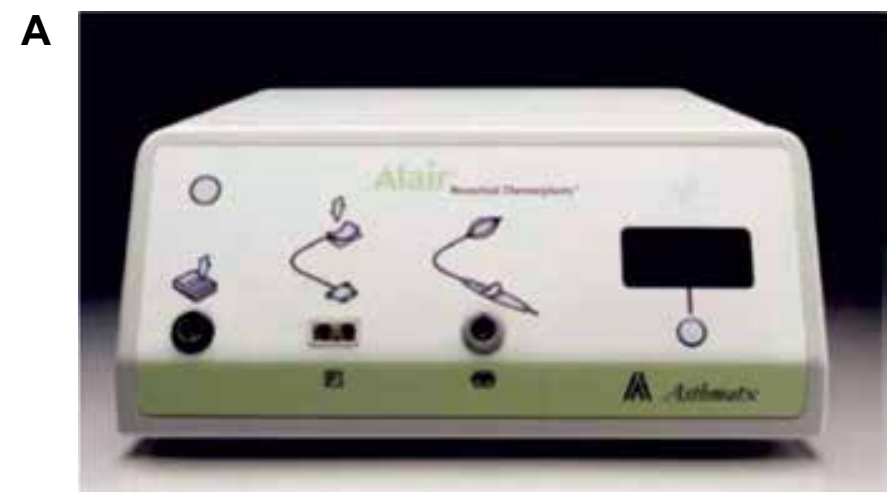

B
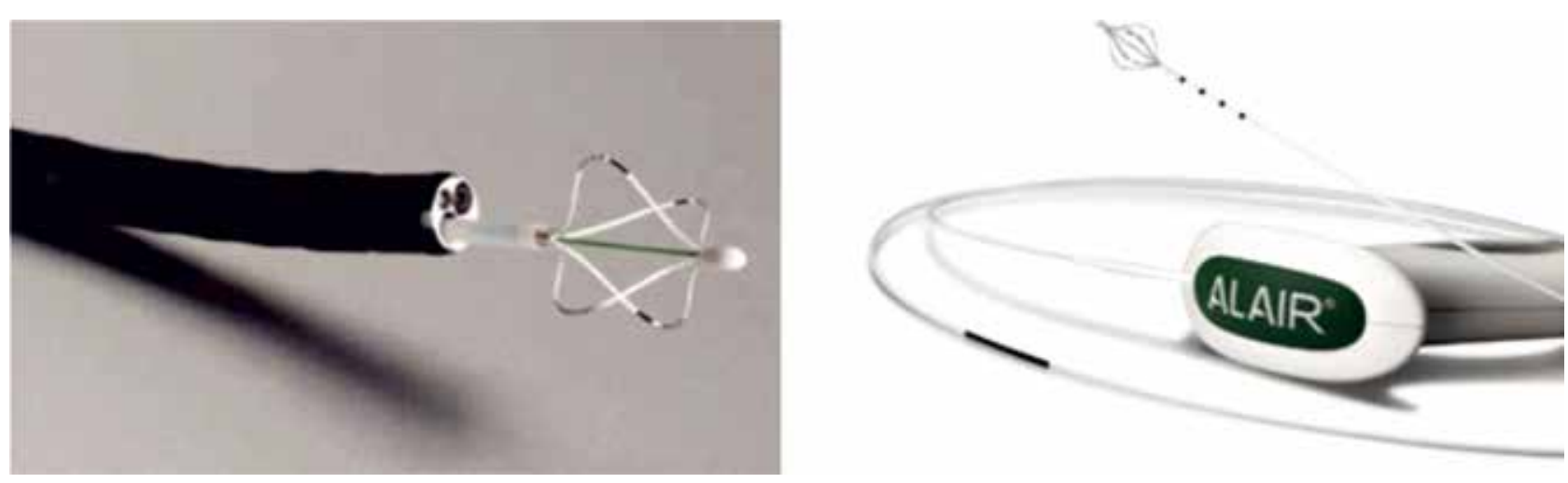

Figura 1. Sistema Alair. A: Controlador de radiofrecuencia. B: Catéter con guía de electrodos expandibles. Image provided courtesy of Boston Scientific Corporation 


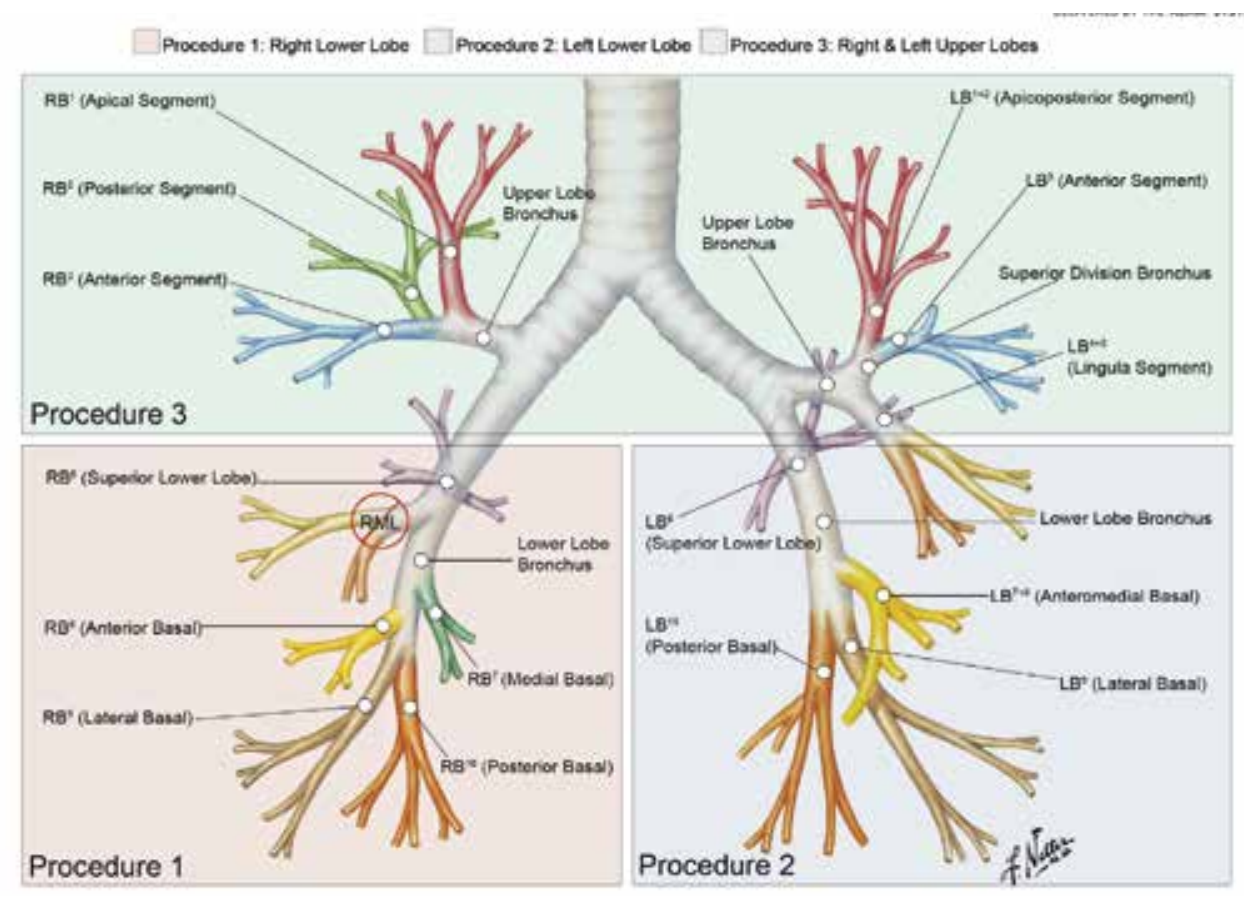

Figura 2. Mapa del procedimiento de termoplastia bronquial. Image provided courtesy of Boston Scientific Corporation

tratamiento. Después de esta exploración se dirige a la región del pulmón que se tratará, y el broncoscopista planifica el orden de los segmentos evitando dejar sin tratar la mayor parte de estos. Se recomienda un enfoque sistemático de distal a proximal; es decir, si se empieza en el lóbulo inferior derecho se puede comenzar con el segmento anterior, seguido de los segmentos lateral y posterior. El segmento medial se trata seguido de la parte distal del bronquio del lóbulo inferior derecho hasta el nivel superior y finalmente el segmento superior. Así mismo, cada segmento también se trata de manera sistemática, de derecha a izquierda. Después de la planificación, se pasa a la región más distal del primer segmento a tratar; el catéter posee marcaciones negras cada $5 \mathrm{~mm}$, se introduce en el canal de trabajo y se hace avanzar hasta la marcación más proximal. Se inicia la entrega de energía presionando y soltando el pedal del controlador, el cual entrega de forma automática durante 10 segundos de acuerdo con los parámetros del tratamiento predefinido. Después de cada activación, el broncoscopista reposiciona proximalmente cada $5 \mathrm{~mm}$ hacia proximal hasta la siguiente activación (figura 3). Los electrodos se expanden (como una canastilla) hasta hacer contacto con bronquios de mayor tamaño. La posición de los electrodos debe hacer referencia a marcas anatómicas debido a que el movimiento relativo entre el broncoscopio y el catéter puede cambiarla. Se recomienda el uso del mapa de las vías respiratorias para planificar y realizar un seguimiento de la progresión del tratamiento para cada sesión. En ocasiones el moco se acumula y se pierde visualización, en cuyos casos el catéter se retrae para limpiar los electrodos, así como para irrigar la vía aérea y succionar las secreciones. Un tratamiento completo consta de 30 a 70 activaciones por lóbulo (de acuerdo con la anatomía específica), no produce ningún efecto macroscópico en la mucosa, 


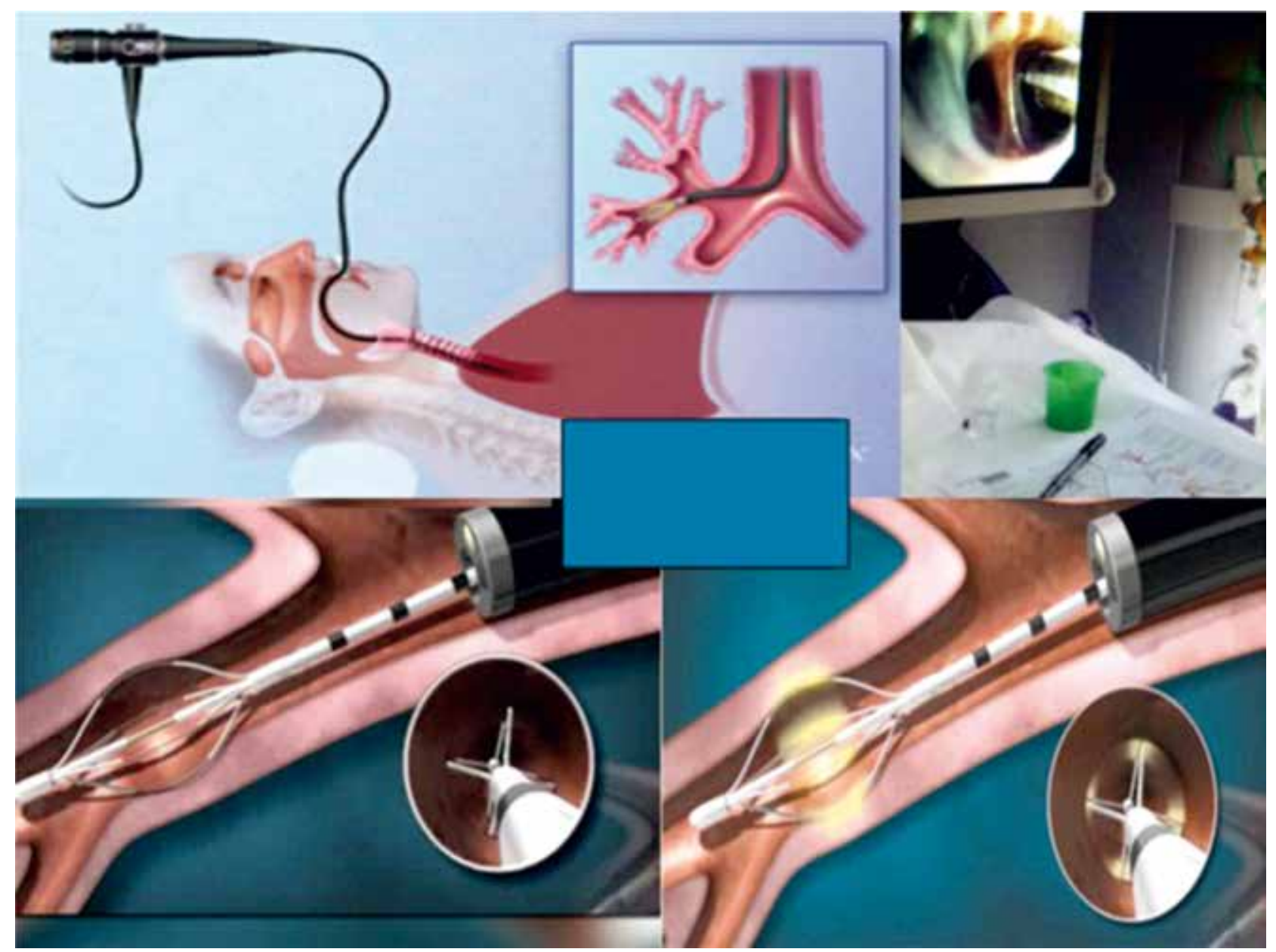

Figura 3. Procedimiento de termoplastia bronquial. A: Esteralizado a través de un broncoscopio. B: Catéter introducido en el bronquio. C: Los electrodos del catéter coaptan en la pared de la vía aérea. D: El catéter libera energía térmica. Image provided courtesy of Boston Scientific Corporation

pero puede provocar blanqueamiento transitorio (3). El procedimiento puede llevarse a cabo bajo sedación o anestesia general.

\section{Estudios clínicos}

El primer estudio clínico de termoplastia bronquial en humanos se llevó a cabo con el objetivo de determinar la seguridad del procedimiento en ocho pacientes programados para resección pulmonar por sospecha o diagnóstico de cáncer pulmonar (11). Todos toleraron el procedimiento y se sometieron a la cirugía. La termoplastia bronquial se estudió por primera vez en 16 asmáticos con asma leve a moderada. Todos tuvieron un control del asma sin infección reciente o exacerbación de los síntomas, con lo cual se demostró que era viable llevar a cabo la termoplastia bronquial de forma segura. Algunos parámetros como el número de días libres de síntomas y el pico flujo espiratorio, mostraron mejoría luego de tres meses; sólo un paciente recibió dos tratamientos debido a infecciones recurrentes (12). Después de este estudio inicial se hicieron tres ensayos clínicos aleatorizados y controlados con asma moderada a grave (13-15): el estudio de intervención en asma (AIR), el estudio de investigación en asma grave RISA y el estudio de intervención en asma 2 (AIR2) (tabla 1). 


\section{Estudio AIR}

Este fue un estudio controlado, aleatorizado, llevado a cabo en 112 pacientes asmáticos de 18 a 65 años, 56 de los cuales se trataron con termoplastia bronquial y 56 con terapia convencional con esteroides inhalados y beta agonistas de larga acción. El desenlace principal fue la frecuencia de exacerbaciones leves durante el periodo de suspensión de los esteroides inhalados. La gravedad del asma estuvo indicada por el tratamiento. Todos utilizaron 200 (mcg) de esteroides inhalados para mantenimiento y beta agonistas de larga acción. Los resultados primarios se midieron respecto a la tasa de exacerbaciones, caracterizadas por disminución del flujo espiratorio pico tomado en la mañana, incremento de medicamentos de rescate y síntomas nocturnos, ocurridos durante los periodos de retirada de los beta agonistas de larga acción. Los resultados mostraron mejorías en las tasas de exacerbaciones leves en un año, calidad de vida, uso de medicación y número de días libres de síntomas, en pacientes con asma grave en cuanto a ingresos hospitalarios por incremento de sus síntomas. Hubo seis hospitalizaciones en el grupo de termoplastia bronquial y dos en el de terapia estándar. Los cambios en $\mathrm{VEF}_{1}$ y la hiperreactividad bronquial, no fueron estadísticamente significativos pero sí se demostró mejoría en los días libres de síntomas y en los cuestionarios de calidad de vida de los pacientes (14).

\section{Estudio RISA}

Se trató de un estudio controlado, aleatorizado, en el que incluyeron 32 pacientes con asma grave no controlada a pesar de dosis altas de esteroides inhalados, beta agonistas de larga acción, antagonistas de los leucotrienos o prednisona oral hasta $30 \mathrm{mg}$ /día. 15 pacientes se trataron con termoplastia bronquial y 17 continuaron la terapia convencional. Después de la evaluación inicial, se retiró la terapia con esteroides entre las semanas 22 y 36 donde se evaluaron, documentándose mejoría en el control del asma y en el flujo pico. Se redujo la dosis de esteroides y aunque no hubo diferencia estadística, la disminución de esteroides fue mayor en el grupo tratado con termoplastia bronquial. A 52 semanas de estudio, el grupo de termoplastia bronquial permaneció con mejoría de su control indicada por menor necesidad de broncodilatadores de rescate y mejoría reportada en los cuestionarios de calidad de vida (puntaje AQLQ) y en el cuestionario de control del asma (ACQ) (13).

\section{AIR2}

En este estudio, la aprobación de termoplastia bronquial para el tratamiento del asma por la FDA se basó en la reducción de las exacerbaciones graves de asma. Es el único doble ciego, aleatorizado controlado por simulación de termoplastia bronquial en pacientes con asma no controlada, a pesar de dosis altas de esteroides inhalados y beta

Tabla 1. Comparación de los criterios de inclusión de los ensayos clínicos de termoplastia bronquial en asma

\begin{tabular}{|c|c|c|c|c|}
\hline Característica & Viabilidad & AIR & RISA & AIR2 \\
\hline Edad (años) & $18-65$ & $18-65$ & $18-65$ & $18-65$ \\
\hline $\begin{array}{l}\text { Dosis de esteroide inhalado (mcg) } \\
\text { (beclometasona o equivalente }\end{array}$ & $\leq 1800$ & $\geq 200$ & $>1500$ & $>1000$ \\
\hline $\begin{array}{l}\text { Dosis de beta agonistas de larga } \\
\text { acción (mcg) }\end{array}$ & $0-100$ & $\geq 100$ & $\geq 100$ & $\geq 100$ \\
\hline Esteroides orales (mg) & 0 & 0 & $<30$ & $<10$ \\
\hline Prebroncodilatador $\mathrm{VEF}_{1}{ }^{*}$ & $\begin{array}{l}>65 \text { (postermoplastia } \\
\text { bronquial) }\end{array}$ & $60-85$ & $\geq 50$ & $\geq 60$ \\
\hline
\end{tabular}


agonistas de larga acción. Es el mayor estudio realizado para termoplastia bronquial, y en él se incluyeron 190 pacientes que fueron tratados con termoplastia bronquial y 98 que recibieron simulación de termoplastia. Este último tratamiento, reproduce todas las señales de audio y visuales de la técnica, pero el catéter no libera energía de radiofrecuencia. El tratamiento fue hecho por un equipo de broncoscopia no cegado, en tanto que todas las evaluaciones y consultas de seguimiento se llevaron a cabo por un equipo cegado. El resultado final del estudio fue la mejoría en los cuestionarios de calidad de vida; no obstante, se ha generado controversia y confusión acerca de la verdadera eficacia de la termoplastia bronquial para el asma grave ya que las tasas de exacerbaciones de asma, días libres de síntomas, uso de medicamento de rescate, visitas a urgencias y hospitalizaciones fueron diferentes entre los grupos (15).

\section{Estudios de imagen}

Algunos autores han evaluado la tomografía de tórax para estudiar los efectos de la termoplastia bronquial en el calibre bronquial, la sensibilidad bronquial a la metacolina y la respuesta a la inspiración profunda (16). Las mediciones tomadas a cinco semanas de realizada la termoplastia bronquial, sugirieron disminución de la hiperreactividad bronquial sin cambios en la distensibilidad pulmonar. En el estudio de viabilidad, se evaluaron 16 pacientes con tomografía de tórax de alta resolución al inicio, a un año y a dos años del estudio, sin encontrar consistencia en la presencia de bronquiectasias o engrosamiento de la pared bronquial o en el parénquima pulmonar (12). Los resultados a 5 años en 93 pacientes del estudio AIR2, no mostraron cambios en el $82 \%$ de los casos. En tres pacientes (3\%) se documentó empeoramiento o formación de nuevas bronquiectasias (15). No hay reportes de patrón en mosaico que puedan sugerir bronquiolitis, estenosis bronquial o enfisema.

\section{Seguridad del procedimiento}

Para los cuatro estudios mencionados se han descrito eventos adversos observados durante la fase de tratamiento (hasta seis semanas después de la última broncoscopia) y la fase de postratamiento (a partir de seis semanas después de la última broncoscopia). Los pacientes tratados con termoplastia bronquial desarrollaron más síntomas respiratorios (tos, sibilancias, expectoración, disnea) que los controles, y ocasionalmente tenían síntomas generales como fiebre en las horas siguientes al tratamiento. Estos síntomas suelen resolverse después de siete días, pero fueron motivo de ingreso en el 3,4\% de los casos con asma moderada a grave en el estudio AIR2 y en el $15,6 \%$ de los casos de asma grave en el estudio RISA. Estos hallazgos resaltan la importancia de optimizar el control del asma antes del primer tratamiento y la vigilancia en los días posteriores al mismo. En el AIR2 un paciente presentó hemoptisis significativa del lóbulo superior derecho un mes después de la última sesión, por lo cual requirió embolización de la arteria bronquial (16). Se observó un sangrado ligero durante el tratamiento de este lóbulo; esta complicación puede ser inherente al tratamiento de termoplastia bronquial (15).

La aplicación de energía térmica controlada $\left(65^{\circ} \mathrm{C}\right)$ plantea si la termoplastia bronquial puede inducir un mayor daño a la pared bronquial. Después del seguimiento de los cuatro estudios, no hubo evidencia de estenosis de la vía aérea o bronquiectasias. Ninguno de los ensayos documentó daño bronquial al primer año de seguimiento. Un paciente en el estudio AIR2 desarrolló un absceso pulmonar catorce meses después del tratamiento; se sometió a resección por cirugía y no se documentó anormalidad bronquial en el estudio histopatológico. Esta complicación se relacionó con una infección respiratoria sin conexión clara con la termoplastia bronquial (16). 


\section{Seguimiento a cinco años}

Durante cinco años de seguimiento, se evaluaron 162 pacientes $(85,3 \%)$ de 190 pacientes del grupo tratado en el estudio AIR2. La proporción de pacientes con exacerbaciones severas y visitas a Urgencias, junto con la cantidad de eventos en un año y cinco postratamiento, se mantuvo baja, menor a la observada doce meses previos al procedimiento. El promedio de reducción de proporciones a cinco años, fue de $44 \%$ para las exacerbaciones y de $78 \%$ para las visitas a Urgencias. No hubo cambios en eventos adversos respiratorios y hospitalizaciones entre el año 2 y año 5 postermoplastia (17). El volumen espiratorio forzado al primer segundo $\left(\mathrm{VEF}_{1}\right)$ se mantuvo estable en los dos estudios con asma moderada a grave (18); adicionalmente no se observaron cambios en la radiografía de tórax ni en la tomografía de tórax de alta resolución a los tres y cinco años, excepto anormalidades transitorias y temporales que suelen verse en pacientes con asma moderada a grave (17). En el estudio RISA el seguimiento de las radiografías de tórax a los cinco años se llevó a cabo en un pequeño grupo de pacientes con asma grave sin cambios significativos; el $\mathrm{VEF}_{1}$ no se deterioró y el número de hospitalizaciones por exacerbación del asma habían disminuido durante este tiempo (12).

\section{Selección de pacientes}

Los estudios clínicos han abordado una amplio rango de gravedad de los pacientes con asma, de leve a severa, y se han hecho, aunque no de manera exclusiva en casos con asma no controlada. Puede haber una tendencia a reservar la termoplastia bronquial para los pacientes con asma no controlada a pesar de dosis altas de esteroide inhalado y beta agonistas de larga acción. La dosis diaria de esteroide inhalado para considerar la termoplastia bronquial no está del todo clara. Se observaron beneficios en pacientes mal controlados a pesar de una dosis diaria de $750 \mathrm{mg}$ de fluticasona en el estudio RISA (11) y beclometasona de $1.000 \mathrm{mg}$ en el AIR2 (15). Se recomienda optimizar el tratamiento antes de ofrecer la termoplastia bronquial.
En el desarrollo del programa clínico, el $\mathrm{VEF}_{1}$ se consideró como parámetro para la selección del paciente, justificado por el aumento de la incidencia de hospitalización tras la realización de termoplastia bronquial en el estudio RISA de 15,6\% vs. 3,4\% respecto al AIR2. En este último estudio requirieron una espirometría prebroncodilatador del $60 \%$ del predicho vs. $50 \%$ en el RISA para la elegibilidad de los pacientes. Se deduce, entonces, que la termoplastia bronquial está indicada en asma mal controlada o no controlada a pesar del máximo tratamiento médico. El control del asma se puede evaluar de varias maneras entre las que se incluyen la frecuencia de las exacerbaciones y los cuestionarios como el del control del asma y de calidad de vida basado en los síntomas diarios. De otro lado, es posible que no sea imprescindible contar con historia de exacerbaciones graves para beneficiarse de la terapia. Los pacientes deben ser seleccionados por un equipo médico conformado por un especialista y un broncoscopista experimentado, y cumplir los siguientes criterios:

- Control en tratamiento farmacológico de acuerdo con las guías de práctica clínica $(9,10)$.

- Estabilidad del asma (ausencia de infección del tracto respiratorio, exacerbación del asma en las últimas cuatro semanas, $\mathrm{VEF}_{1}$ dentro del $10 \%$ del mejor valor.

- Ausencia de comorbilidad que ponga en riesgo el procedimiento; por ejemplo: apnea obstructiva del sueño no tratada o enfermedad cardiovascular clínicamente significativa, epilepsia, diabetes insulinodependiente o cáncer.

Los pacientes con asma que pone en riesgo la vida se excluyeron de los estudios. Del mismo modo, se tienen en cuenta tres o más admisiones hospitalarias o cuatro o más ciclos de esteroides sistémicos en el año anterior a la termoplastia (15, 16). El uso de broncodilatadores de corta acción (mayor a cuatro inhalaciones por día) también fue un criterio de exclusión para el AIR2, así como pacientes con tres o más antecedentes de infecciones respiratorias probadas tratadas con antibióticos el año anterior. 
Las advertencias y precauciones actuales y exclusiones son: enfermedad pulmonar obstructiva crónica, bronquiectasias, infecciones respiratorias recurrentes o cualquier otra enfermedad respiratoria significativa no controlada, anticoagulación, marcapasos y desfibrilador. Se excluyen, así mismo, personas con un índice tabáquico de 10 o más (15) para tratamiento. La termoplastia bronquial puede incluso llevar a un empeoramiento transitorio de los síntomas del asma, la intensidad de los cuales puede ser proporcional a la gravedad del paciente.

\section{Tratamiento con esteroides sistémicos}

Al final del tratamiento (seis semanas después de la última broncoscopia), es ideal revaluar el beneficio clínico. Los tratamientos farmacológicos se ajustan a las dosis más bajas posibles, comenzando con una reducción de esteroides orales seguida por una disminución de esteroides inhalados y beta agonistas de larga acción. No existen datos sobre los efectos de la termoplastia bronquial en la inflamación de las vías respiratorias para guiar empíricamente el tratamiento farmacológico ni los efectos del retiro del mismo (15).

\section{Base de datos Cochrane}

Una revisión sistemática realizada en 2014 por Torrego et al. (20), incluyó tres estudios clínicos con un total de 429 pacientes. El estudio encontró mejoría en la calidad de vida a los 12 meses en los que se utilizó la termoplastia bronquial, pero no alcanzó significancia estadística; en relación con control de los síntomas tampoco mostró significancia estadística. El sesgo puede ser alto debido a que los primeros estudios no tenían la simulación realizada en el AIR2, en el que se mostró una menor tasa de exacerbaciones e ingresos a urgencias después de doce meses de tratamiento. Los estudios no mostraron mejoría significativa en los parámetros de función pulmonar (a excepción de un mayor aumento en el flujo espiratorio máximo matutino). No observaron diferencia significativa en el riesgo de hospitalización al final del periodo de tratamiento. Se asoció con un aumento de eventos respiratorios principalmente durante el periodo del tratamiento, la mayoría de los cuales fueron de intensidad leve o moderada, aparecieron en el periodo postratamiento de 24 horas y se resolvieron en una semana. Los autores concluyeron que la termoplastia bronquial en pacientes con asma moderada a grave, ofrece un beneficio clínico modesto en la calidad de vida y menores tasas de exacerbación del asma, sin diferencias significativas en las puntuaciones de control de la enfermedad. Existe sesgo ya que se observaron los principales beneficios en los dos estudios que no incluían el tratamiento simulado (AIR2). La termoplastia bronquial aumenta el riesgo de eventos adversos durante el tratamiento, pero tiene un perfil de seguridad razonable después de terminado. Para la práctica clínica sería aconsejable recopilar datos de los pacientes de manera sistemática en los registros clínicos independientes. Se requieren investigaciones futuras para proporcionar una mejor comprensión de los mecanismos de acción de la termoplastia bronquial, así como su efecto en diferentes fenotipos de asma o en pacientes con peor función pulmonar (19).

\section{Futuras investigaciones}

Aunque no existen datos de efectos adversos importantes de la termoplastia bronquial en un plazo de cinco años, se desconocen las consecuencias a largo plazo. Por tanto, se requieren estudios diseñados para determinar sus efectos sobre los resultados primarios relevantes, tales como las tasas de exacerbación, y sobre los efectos a largo plazo en la función pulmonar. También se necesitan estudios para entender con mayor claridad los fenotipos de los pacientes que responden mejor, los efectos en aquellos con asma grave $\left(\mathrm{VEF}_{1}\right.$ menor al $60 \%$ del valor predicho) o quienes se utilizan esteroides sistémicos, sus beneficios y seguridad a largo plazo. Estas investigaciones adicionales probablemente tengan un impacto considerable en las recomendaciones 
futuras de la termoplastia bronquial. Adicionalmente, los mecanismos de acción y la selección óptima de los pacientes deben ser abordados en futuros estudios clínicos y científicos rigurosos.

\section{Conclusiones}

El uso actual de la termoplastia bronquial va dirigido a pacientes con asma grave no controlada, con dependencia a esteroides, exacerbaciones frecuentes, $\mathrm{VEF}_{1}$ disminuido con pobre calidad de vida. El procedimiento debe ser realizado por un broncoscopista experimentado en conjunto con un equipo multidisciplinario especializado en asma. Los estudios clínicos han demostrado la mejoría de los síntomas, disminución de las exacerbaciones graves y disminución del uso de los internamientos hospitalarios. La experiencia clínica ha demostrado que la termoplastia bronquial es un procedimiento seguro y bien tolerado el cual ofrece muchos beneficios a pacientes con asma grave no controlada. Estos beneficios se mantienen por un período mínimo de por lo menos 5 años.

\section{Bibliografía}

1. FDA approves new device for adults with severe and persistent asthma. US Food and Drug Administration website. Disponible en: http://www. fda.gov/NewsEvents/Newsroom/ PressAnnouncements/ ucm209909.htm. April 27, 2010. [Acceso 10 Apr 2014].

2. Ebina M, Takahashi T, Chiba T, Motomiya M. Cellular hypertrophy and hyperplasia of airway smooth muscles underlying bronchial asthma: a 3-D morphometric study. Am Rev Respir Dis. 1993;148:720-6.

3. Mayse ML, Laviolette M, Rubin AS. Clinical pearls for bronchial thermoplasty. J Bronchol. 2007; 14:115-23.

4. Cox PG, Miller J, Mitzner W, Leff AR. Radiofrequency ablation of airway smooth muscle for sustained treatment of asthma: preliminary investigations. Eur Resp J. 2004;24:659-63.

5. Jeffery PK. Remodeling in asthma and chronic obstructive lung disease. Am J Respir Crit Care Med. 2001;164:S28-38.

6. Brown RH, Wizeman W, Danek C, Mitzner W. In vivo evaluation of the effectiveness of bronchial thermoplasty with computed tomography. J Appl Physiol. 2005;98:1603-6.

7. Dyrda P, Tazzeo T, DoHarris L, Nilius B, Roman HN, Lauzon AM, Aziz T, Lukic D, Janssen LJ. Acute response of airway muscle to extreme temperature includes disruption of actin-myosin interaction. Am J Respir Cell Mol Biol. 2011;44:213-22.

8. Cox G, Miller JD, McWilliams A, Fitzgerald JM, Lam S. Bronchial thermoplasty for asthma. Am J Respir Crit Care Med. 2006;173:965-9.

9. Global Initiative for Asthma: Global Strategy for Asthma Management and Prevention. Revised December 2011. Disponible en: http://www.ginasthma.com/Guidelineitem. asp? $11=2 \& 12=1 \&$ intId $=60$

10. Chung KF, Wenzel SE, Brozek JL, Bush A, Castro M, Sterk $\mathrm{PJ}$, et al. International ERS/ATS guidelines on definition, evaluation and treatment of severe asthma. Eur Respir J. 2014;43:343-73.

11. Miller JD, Cox G, Vincic L, Lombard CM, Loomas BE, Danek CJ. A prospective feasibility study of bronchial thermoplasty in the human airway. Chest. 2005;127:1999-2006.

12. Wahidi MM, Kraft M. Bronchial thermoplasty for severe asthma. Am J Respir Crit Care Med. 2012 Apr 1;185:709-14.

13. Pavord ID, Cox G, Thomson NC, Rubin AS, Corris PA, Niven RM, et al. Safety and efficacy of bronchial thermoplasty in symptomatic, severe asthma. Am J Respir Crit Care Med. 2007;176:1185-91.

14. Cox G, Thomson NC, Rubin AS, Niven RM, Corris PA, Siersted $\mathrm{HC}$, et al. Asthma control during the year after bronchial thermoplasty. N Engl J Med. 2007;356:1327-37.

15. Castro M, Rubin AS, Laviolette M, Fiterman J, De Andrade Lima M, et al. Effectiveness and safety of bronchial thermoplasty in the treatment of severe asthma: a multicenter, randomized, double-blind, sham-controlled clinical trial. Am J Respir Crit Care Med. 2010; 181:116-24.

16. Castro M, Rubin A, Laviolette M, Hanania NA, Armstrong B, Cox G; AIR2 Trial Study Group. Persistence of effectiveness of bronchial thermoplasty in patients with severe asthma. Ann Allergy Asthma Immunol. 2011;107:65-70.

17. Wechsler ME, Laviolette M, Rubin AS, Fiterman J, Lapa e Silva JR, Shah PL, et al. Bronchial thermoplasty: long-term safety and effectiveness in patients with severe persistent asthma. J Allergy Clin Immunol. 2013;132:1295-302.

18. Pavord ID, Thomson NC, Niven RM, Corris PA, Chung KF, Cox G, et al. Research in Severe Asthma Trial Study Group. Safety of bronchial thermoplasty in patients with severe refractory asthma. Ann Allergy Asthma Immunol. 2013;111:402-7.

19. Wechsler ME, Laviolette M, Rubin AS, Fiterman J, Lapa e Silva JR, Shah PL, et al. Bronchial thermoplasty: long-term safety and effectiveness in patients with severe persistent asthma. J Allergy Clin Immunol. 2013;132:1295-302.

20. Torrego A, Sola I, Muñoz A. Bronchial thermoplasty for moderate or severe persistent asthma in adults. Cochrane Database Syst Rev. 2014. 